\title{
INTEGRABLE SYSTEMS IN SYMPLECTIC GEOMETRY
}

\author{
E. ASADI* and J. A. SANDERS \\ Department of Mathematics, Faculty of Sciences, Vrije Universiteit, Amsterdam, The Netherlands
}

\begin{abstract}
Quaternionic vector mKDV equations are derived from the Cartan structure equation in the symmetric space $\mathbb{M} \mathbb{P}^{n}=S p(n+1) / S p(1) \times S p(n)$. The derivation of the soliton hierarchy utilizes a moving parallel frame and a Cartan connection 1 -form $\omega$ related to the Cartan geometry on $\mathbb{M P}^{n}$ modelled on $\left(\mathfrak{s p}_{n+1}, \mathfrak{s p}_{1} \times \mathfrak{s p}_{n}\right)$. The integrability structure is shown to be geometrically encoded by a PoissonNijenhuis structure and a symplectic operator.
\end{abstract}

1. Introduction. Many of the equations and systems which now are called integrable have been known in differential geometry. One of them is the famous sine-Gordon equation (SG), which was derived to describe surfaces with constant negative Gaussian curvature. Another one is the Liouville equation describing minimal surfaces in three-dimensional Euclidean space. For physicists, the prototype examples of integrable systems are the Korteweg-De Vries equation (KDV) and the nonlinear Schrödinger (NLS) equation.

The connection between geometry and integrable systems was clarified by Hasimoto [6] in 1972. He found the transformation between the equations governing the curvature and torsion of a thin vortex filament (FM) moving in an incompressible inviscid fluid and the NLS equation. The equation FM can be modelled as

$$
\gamma_{t}=\gamma_{s} \times \gamma_{s s}
$$

in which $\gamma(s, t)$ is a curve evolving in three-dimensional space $\mathbb{R}^{3}$. In fact Hasimoto constructed the complex function $\psi=\kappa \exp \left(i \int_{0}^{s} \tau d s\right)$ of the curvature and torsion of the curve $\gamma$, and showed that if the curve evolves according to the FM equation, then $\psi$ solves the cubic NLS equation

$$
i \psi_{t}+\psi_{s s}+\frac{1}{2}|\psi|^{2} \psi=0
$$

Doliwa and Santini [4] showed that certain elementary geometric properties of the motion of a curve select the hierarchy of integrable dynamics. The motion should be non-stretching and occur in a $N$-dimensional sphere of radius $R$ and the dynamics independent of the radius of the sphere. This gives a simple geometric meaning to the Hasimoto transformation: the Hasimoto transformation is (induced by) a gauge transformation from the Frenêt frame to the parallel or natural frame.

Generalizing Doliwa and Santini's approach, Sanders, Wang and Beffa [11] showed that motion of a curve in a three-dimensional Riemannian manifold with constant curvature follows an arc-length preserving geometric evolution and the evolution of its curvature and torsion is always a Hamiltonian flow.

${ }^{*}$ We thank $\mathcal{N} \mathcal{W O}$ (Netherlands Organization for Scientific Research) for financial support of the project Geometry and classification of integrable systems. 
Given the Cartan connection 1-form $\omega$ defined on the geometric flow $\gamma$ on a Riemannian manifold, a gauge transformation on $\omega$ yields the new connection

$$
\tilde{\omega}=A d\left(h^{-1}\right) \omega+h^{-1} d h .
$$

Hence the Frenêt frame encoded in Cartan connection matrix $\omega\left(\gamma_{s}\right)$ would transform to $\tilde{\omega}\left(\gamma_{s}\right)$.

Cartan's Lemma associates the Lie algebra valued 1-form, the Cartan connection, to the Levi-Civita connection defined on a Riemannian manifold, so that having a frame on the curve embedded in the Riemannian manifold is equivalent to specifying the Cartan connection applied on the $\gamma_{s}$. Indeed Sanders and Wang $[13,14]$ showed that choosing a natural frame and having the Cartan connection specified according to the natural frame, the Cartan structure equation leads to the recursion equation of integrable equation. In this way they found the Hamiltonian operator out of the curvature part and the symplectic operator resulted from solving the free torsion tensor. Moreover, the flow given by the trivial symmetry, yields the vector mKDV evolution equation.

The present paper results from a generalization of the Riemannian to the symplectic case. The research was done by the first author, supported by $\mathcal{N} \mathcal{W O}$, under supervision of the second and published in [3].

We obtain the geometric operators such as Hamiltonian, symplectic and Nijenhuis operators of the soliton equation encoded as

$$
\hat{u}_{t}=\hat{\mathfrak{H}} \hat{\mathfrak{I}} \hat{m}_{0}+\hat{\mathfrak{A}} \hat{m}_{0}
$$

arising from Cartan structure equation in symplectic geometry which is encoded as a Riemannian symmetric space $\mathbb{H}^{n}=S p(n+1) / \operatorname{Sp}(1) \times \operatorname{Sp}(n)$. These operators will be given also in terms of the Lie bracket, Killing form and projections of underlying Lie algebras. More precisely, we prove that the operators $\hat{\mathfrak{H}}$ and $\hat{\mathfrak{A}} \hat{\mathfrak{H}}$ determine the Poisson-Nijenhuis structure [10]. Also the conjugation $\hat{\mathfrak{A}}{ }^{-1 *} \hat{\mathfrak{I}} \hat{\mathfrak{A}}^{-1}$ of the antisymmetric operator $\hat{\mathfrak{I}}$ by the Nijenhuis operator $\hat{\mathfrak{A}}$ will be a symplectic operator. Furthermore, the operator $\hat{\mathfrak{R}}=\hat{\mathfrak{H}} \hat{\mathfrak{I}} \hat{\mathfrak{A}}^{-1}$ will be a recursion operator transforming the symmetry of the soliton equation to another symmetry. The soliton evolution equation can be found by applying the recursion operator $\hat{\mathfrak{R}}$ to the trivial symmetry.

The outline of the paper is as follows. In the next section we explore the basic set up for the Cartan geometry and in particular for the reduction Cartan geometry as well as the homgeneous symmetric space. In Section 3, we study the integrability in Euclidean geometry in a way that generalizes to symplectic geometry in Section 4. In Section 5 we express the evolution equation of the differential invariants encoded in the Cartan matrix in terms of Lie algebraic object as we did in Euclidean geometry. In Section 6, we discuss the Hamiltonian, symplectic and Nijenhuis operators. We will give details of the proof of some of the assertions. The reader can find the missing details in [3].

\section{Geometric preliminaries.}

2.1. Cartan geometry. We generalize the idea of classical Cartan connection. The content of this section can be found in [15]. As in Euclidean space there is a natural way to parallel translate and compare vectors at different points, likewise in 
a general manifold a choice of a connection prescribes a way of translating tangent vectors 'parallel to themselves' and to intrinsically define a directional derivative.

In the case of a principal bundle $P$ with structure group $G$ over a manifold $M$, we explain the rule of a connection when thinking of lifting a vector field $v \in T M$ to a vector field $\tilde{v} \in T P$ in a unique way. For each $p \in P$, let $G_{p}$ be the vector subspace of $T_{p} P$ consisting of all the vectors tangent to the vertical fibre. That is $G_{p}=\operatorname{ker}(d \pi(p)) \subset T_{p} P$ in which $d \pi(p): T_{p} P \rightarrow T_{\pi p} M$.

The lifting of $v$ will be unique if we require $\tilde{v}(p)$ to lie in a subspace of $T_{p} P$ complementary to $G_{p}$. A smooth and $G$-invariant choice of such a complementary subspace is called a Ehresmann connection on $P$. This leads to the following definition.

Definition 1. A connection on a principal bundle $P$ is a smooth assignment of a subspace $H_{p} \subset T_{p} P$, for each $p \in P$ such that:

(1) $T_{p} P=G_{p} \oplus H_{p}$,

(2) $H_{g p}=T_{p}\left(L_{g}\right) H_{p}$ for each $g \in G$, where $L_{g}$ is the left-translation in $G$ and consequently $T_{p}\left(L_{g}\right): T_{p} P \rightarrow T_{g p} P$.

Given a connection, the horizontal subspace $H_{p}$ is mapped isomorphically by $d \pi$ onto $T_{\pi p} M$. Therefore the lifting of $v$ is the unique horizontal $\tilde{v}$ which projects onto $v$. An equivalent way of assigning a connection is by means of a Lie algebra valued 1-form $\omega$ (Cartan connection). If $X \in \mathfrak{g}$, let $X^{\dagger}$ be the vector field on $P$ induced by the action of the 1-parameter subgroup $e^{t X}$. Since the action of $G$ maps each fibre into itself, then $X^{\dagger}$ is tangent to the vertical fibre at each point, i.e., $X \in G_{p}$. For each $v \in T_{p} P$, we define $\omega(v)$ as the unique $X \in \mathfrak{g}$ such that $X^{\dagger}$ is equal to the vertical component of $v$. It follows that $\omega(v)=0$ if and only if $v$ is horizontal.

Proposition 1. A Cartan connection 1-form $\omega$ has the following properties:

(1) $\omega\left(X^{\dagger}\right)=X$,

(2) $L_{g}^{*} \omega=A d_{g} \omega$ for each $g \in G$, in which Ad is adjoint representation of $G$.

The proof can be found in appendix A of [15].

Now we define the Cartan geometry based on the Ehresmann connection. Assume here that $H$ is a group with the Lie algebra $\mathfrak{h}$ as subalgebra of $\mathfrak{g}$.

Definition 2. A Cartan geometry $\xi=(P, \omega)$ on $M$ modelled on $(\mathfrak{g}, \mathfrak{h})$ with group $H$ consist of the following data:

(1) a smooth manifold $M$;

(2) a principal left $H$ bundle $P$ over $M$;

(3) a $\mathfrak{g}$-valued 1 -form $\omega$ on $P$ satisfies the following conditions:

(a) for each point $p \in P$, the linear map $\omega_{p}: T_{p} P \rightarrow \mathfrak{g}$ is an isomorphism;

(b) $\left(L_{h}\right)^{*} \omega=\left(\operatorname{Ad}_{h}\right) \omega$ for all $h \in H$;

(c) $\omega\left(X^{\dagger}\right)=X$ for all $X \in \mathfrak{h}$.

The $\mathfrak{g}$-valued form on $P$ given by

$$
\Omega=d \omega+\frac{1}{2}[\omega, \omega]
$$

is called the curvature. If $\rho: \mathfrak{g} \rightarrow \mathfrak{g} / \mathfrak{h}$ is the canonical projection, then $\rho^{*} \Omega$ is called the torsion. If $\Omega$ takes values in the subalgebra $\mathfrak{h}$, we say that the geometry is torsion free. 
Definition 3. Let $M$ be a connected manifold. Then the Cartan geometry $\xi=$ $(P, \xi)$ has constant curvature if $\Omega_{p}\left(X_{p}, Y_{p}\right)$ is independent of $p \in P$ whenever the vector fields $X$ and $Y$ are $\omega$-constant vector fields.

This may also be expressed by saying that the curvature function

$$
K: P \rightarrow \operatorname{Hom}\left(C^{2}(\mathfrak{g} / \mathfrak{h}), \mathfrak{h}\right), \quad K(p)=\Omega_{p}\left(\omega_{p}^{-1}(u), \omega_{p}^{-1}(v)\right)
$$

is constant.

DeFinition 4. A Cartan geometry whose curvature vanishes at every point is called flat.

Notice that while the structure equation always holds for a Lie group, meaning that the curvature of the Maurer-Cartan form vanishes, not all Cartan geometries are flat.

2.2. Homogeneous space, symmetric space. The material of this section is taken from [2] and [7]. A homogeneous space of a Lie group $G$ is any differentiable manifold $P$ on which $G$ acts transitively, that is, for $p_{1}, p_{2} \in M$, there is $g \in G$ so that $g \cdot p_{1}=p_{2}$. The subgroup

$$
H=H_{p_{0}}=\left\{g \in G: g \cdot p_{0}=p_{0}\right\}
$$

is called the isotropy group at $p_{0}$. It is a theorem that each such $P$ can be identified with a coset space $G / H$ for some subgroup $H$ and that this $H$ plays the rule of isotropy group of some point.

Let $\mathfrak{g}, \mathfrak{h}$ be the Lie algebras of $G$ and $H$, respectively. Then we get canonical isomorphism

$$
\mathfrak{g} / \mathfrak{h} \cong T_{o} G / H
$$

due the fact that $\operatorname{ker} d \pi_{e}=\mathfrak{h}$ where $\pi$ is the projection $\pi: G \rightarrow G / H$.

Now, we will consider the important special case.

Definition 5. When $\mathfrak{g}$ A homogeneous space is called reductive if there exist a subspace $\mathfrak{m}$ of $\mathfrak{g}$ such that $\mathfrak{g}$ satisfies the more stringent conditions:

$$
\mathfrak{g}=\mathfrak{h} \oplus \mathfrak{m}, \quad[\mathfrak{h}, \mathfrak{h}] \subset \mathfrak{h}, \quad[\mathfrak{h}, \mathfrak{m}] \subset \mathfrak{m},
$$

as an immediate consequence of the above isomorphism, if $G / H$ is reductive, we have the canonical isomorphism

$$
\mathfrak{m} \cong T_{o} G / H .
$$

DEFINITION 6. When $\mathfrak{g}$ satisfies the conditions:

$$
\mathfrak{g}=\mathfrak{h} \oplus \mathfrak{m}, \quad[\mathfrak{h}, \mathfrak{h}] \subset \mathfrak{h}, \quad[\mathfrak{h}, \mathfrak{m}] \subset \mathfrak{m}, \quad[\mathfrak{m}, \mathfrak{m}] \subset \mathfrak{h},
$$

then $\mathfrak{g}$ is called a symmetric algebra and $G / H$ is a symmetric space.

If $G / H$ is symmetric space, then the Riemannian metric is the scalar product on $\mathfrak{m}$, so that the vector subspace $\mathfrak{m}$ will be the orthogonal complement of $\mathfrak{h}$ in the direct $\operatorname{sum} \mathfrak{g}=\mathfrak{h} \oplus \mathfrak{m}$. 
REMARK 1. On p. 518 of Helgason's book [7] there is a table of symmetric spaces. Directly beneath this table those spaces which are Hermitian are listed.

REMARK 2. The space $S p(n+1) / S p(n) \times S p(1)$ is a homogeneous space. There we have that $\mathfrak{m}=\mathfrak{g} / \mathfrak{h}$ in which $\mathfrak{g}=\mathfrak{s p}(n+1)$ and $\mathfrak{h}=\mathfrak{s p}(n) \times \mathfrak{s p}(1)$. Moreover this space is naturally reductive space. See [2] for the pertinent definitions.

3. Integrable system in Euclidean geometry. One can start with the Euclidean Lie algebra $\mathfrak{g}=\mathfrak{e u c}_{n}(\mathbb{R})=\mathfrak{o}_{n}(\mathbb{R}) \ltimes \mathbb{R}^{n}$ and $\mathfrak{h}=\mathfrak{o}_{n}$ and assume that $\omega$ is the Cartan connection in Euclidean geometry as a Cartan geometry. Let us denote $\mathfrak{m}=\mathfrak{g} / \mathfrak{h}$ so that we can decompose the Lie algebra $\mathfrak{g}=\mathfrak{h} \oplus \mathfrak{m}$ is direct sum of two vector space $\mathfrak{h}$ and $\mathfrak{m}$. Every element of $\mathfrak{g}$ can be expressed as

$$
\left(\begin{array}{cc}
\mathbf{A} & \mathfrak{b} \\
0 & 0
\end{array}\right), \quad \mathbf{A} \in \mathfrak{o}_{n}, \mathbf{b} \in \mathbb{R}^{n},
$$

and any matrix $\mathbf{A} \in \mathfrak{o}_{n}$ satisfying $\mathbf{A}+\mathbf{A}^{t}=0$. One finds that

$$
[\mathfrak{h}, \mathfrak{h}] \subset \mathfrak{h}, \quad[\mathfrak{h}, \mathfrak{m}] \subset \mathfrak{m} \quad \text { and } \quad[\mathfrak{m}, \mathfrak{m}] \subset \mathfrak{h} .
$$

The Cartan connection applied on $D_{x}$ is chosen so that $\omega\left(D_{x}\right)$ as an element of subalgebra $\mathfrak{g}$ will be moving frame matrix. If we choose the moving frame to be the parallel moving frame, then we have that

$$
\omega\left(D_{x}\right)=\left(\begin{array}{ccc}
0 & -\mathbf{k}^{t} & 1 \\
\mathbf{k} & 0 & 0 \\
0 & 0 & 0
\end{array}\right) .
$$

The choice of column vector $\left(\begin{array}{l}1 \\ 0\end{array}\right)$ is related to the choice of $D_{x}$ as first basis element of the vector space $\mathfrak{g} / \mathfrak{h}$.

Now $\omega\left(D_{t}\right)$ will be a general element of $\mathfrak{g}$. Thus let it be as follows

$$
\omega\left(D_{t}\right)=\left(\begin{array}{ccc}
0 & -\mathbf{m}^{t} & h_{1} \\
\mathbf{m} & \mathbf{M} & \mathbf{h} \\
0 & 0 & 0
\end{array}\right) .
$$

Now we write down the Cartan structure equation

$$
\Omega\left(D_{x}, D_{t}\right)=D_{x} \omega\left(D_{t}\right)-D_{t} \omega\left(D_{x}\right)+\left[\omega\left(D_{t}\right), \omega\left(D_{x}\right)\right],
$$

assuming that $\left[D_{x}, D_{t}\right]=0$. Also we assume that the manifold which modelled on Euclidean geometry is flat. That gives us $\Omega\left(D_{x}, D_{t}\right)=0$, so that Cartan structure equation restrict to the following form:

$$
D_{x} \omega\left(D_{t}\right)-D_{t} \omega\left(D_{x}\right)+\left[\omega\left(D_{t}\right), \omega\left(D_{x}\right)\right]=0 .
$$

Replacing the $\omega\left(D_{x}\right)$ and $\omega\left(D_{t}\right)$ into the Cartan structure equation above would leads to the following equations:

$$
\begin{aligned}
& \mathfrak{h} \text { part }:\left\{\begin{array}{l}
D_{x} \mathbf{m}-D_{t} \mathbf{k}+\mathbf{M} \mathbf{k}=0, \\
D_{x} \mathbf{M}+\mathbf{k m}^{t}-\mathbf{m k}^{t}=0
\end{array}\right. \\
& \mathfrak{g} / \mathfrak{h} \quad \text { part }:\left\{\begin{array}{l}
D_{x} h_{1}+\langle\mathbf{k}, \mathbf{h}\rangle=0 \\
D_{x} \mathbf{h}-\mathbf{k} h_{1}+\mathbf{m}=0
\end{array}\right.
\end{aligned}
$$


Hence we have that

$$
D_{t} \mathbf{k}=\mathfrak{H} \mathbf{m}, \quad \mathbf{m}=\mathfrak{I} \mathbf{h}
$$

where

$$
\mathfrak{H}=D_{x}+\mathfrak{H}_{1}, \quad \mathfrak{I}=-D_{x}-\mathbf{k} D_{x}^{-1}\langle\mathbf{k}, .\rangle,
$$

and

$$
\mathfrak{H}_{1} \mathbf{m}=D_{x}^{-1}\left(\mathbf{m} \mathbf{k}^{t}-\mathbf{k} \mathbf{m}^{t}\right) \mathbf{k} .
$$

Here $\langle.,$.$\rangle denotes the standard inner product on \mathbb{R}^{n-1}$, and $D_{x}^{-1}$ in (4), acts simply on the matrix $\mathbf{m k}^{t}-\mathbf{k m}^{t} \in \mathfrak{o}_{n-1}(\mathbb{R})$ and then the matrix $D_{x}^{-1}\left(\mathbf{m} \mathbf{k}^{t}-\mathbf{k m}^{t}\right) \in \mathfrak{o}_{n-1}(\mathbb{R})$ act on the vector $\mathbf{k} \in \mathbb{R}^{n-1}$ as usual.

In [13], it is proved that $\mathfrak{H}$ and $\mathfrak{I}$ are compatible Hamiltonian and symplectic operators, respectively and $\mathfrak{R}=\mathfrak{H} \mathfrak{I}$ is an hereditary recursion operator. If we choose $\mathbf{h}$ to be the trivial symmetry $\mathbf{k}_{x}$, then we obtain the following vector mKDV evolution equation

$$
\mathbf{k}_{t}=-\mathbf{k}_{3 x}-\frac{3}{2} \mathbf{k}_{x}\langle\mathbf{k}, \mathbf{k}\rangle
$$

One can write the geometric operators $\mathfrak{H}$ and $\mathfrak{I}$ in terms of the Lie bracket, Killing form and projections as follows:

$$
\mathfrak{H}=D_{x}+a d_{u} D_{x}^{-1} \pi_{0} a d_{u}, \quad \mathfrak{I}=-D_{x}-\frac{1}{2} u D_{x}^{-1} K(u, .),
$$

where $u$ is the projection of $\omega\left(D_{x}\right)$ onto $\mathfrak{h}$ and $\pi_{0}$ is a projection defined on $\mathfrak{h}=\mathfrak{o}_{n}(\mathbb{R}) \ltimes 0$ onto $\mathfrak{o}_{n-1}(\mathbb{R}) \ltimes 0$, i.e.,

$$
\pi_{0}\left(\begin{array}{ccc}
0 & -\mathbf{m}^{t} & 0 \\
\mathbf{m} & \mathbf{M} & 0 \\
0 & 0 & 0
\end{array}\right)=\left(\begin{array}{ccc}
0 & 0^{t} & 0 \\
0 & \mathbf{M} & 0 \\
0 & 0 & 0
\end{array}\right)
$$

4. Integrable system in symplectic geometry. We define the symplectic Lie group over a quaternionic algebra by

$$
G=\operatorname{Sp}(n+1)=\left\{A \in G L(n+1, \mathbb{H}) \mid A^{*} A=I\right\},
$$

in which $A_{i j}^{*}=\overline{A_{j i}}, A_{i j} \in \mathbb{H}$. Then the Lie subgroup $H=\operatorname{Sp}(1) \times \operatorname{Sp}(n)$ of $G$ will be closed so that the pair $(G, H)$ will define a Klein geometry. The Lie algebras of $G$ and $H$ are

$$
\mathfrak{g}=\mathfrak{s p}_{n+1}=\left\{A \in G L(n+1, \mathbb{U}) \mid A^{*}+A=0\right\}, \quad \mathfrak{h}=\mathfrak{s p}_{1} \times \mathfrak{s p}_{n}
$$

As is known in the literature, $\mathrm{M}=G / H$ is then a smooth manifold. In fact $\mathrm{M}$ is the quaternionic projective space $\because \mathbb{P}^{n-1}$. For a comprehensive reference, see [15]. Similar to the situation in Riemannian manifold [13], given a curve in $\mathrm{M}$, we know its tangent vectors $D_{x}$ and want to compute all possible $D_{t}$. Let $\omega$ be Cartan 1-form with its values in the Lie algebra $\mathfrak{g}$. We make a specific choice of $\omega\left(D_{x}\right)$ and leave $\omega\left(D_{t}\right)$ as a general 
element of $\mathfrak{g}$. We see that the dimension of $M$ is equal to the dimension of $\mathfrak{g} / \mathfrak{h}$ which is $4 n$. With $x$ taken to be the arc length, the dimension of the space of differential invariants in $\omega\left(D_{x}\right)$ describing the curve must be one less than the dimension of the manifold, that is, $4 n-1$.

Now let us choose a Cartan matrix $\omega\left(D_{x}\right)$ similar to that of the parallel coframe in Riemannian geometry with proper dimension counting as follows:

$$
\omega\left(D_{x}\right)=\left(\begin{array}{ccc}
0 & 1 & \mathbf{0}^{t} \\
-1 & u & -\overline{\mathbf{u}}^{t} \\
\mathbf{0} & \mathbf{u} & \mathbf{0}
\end{array}\right),
$$

where $\omega\left(D_{x}\right)$ is taken as an element of $\mathfrak{s p}(n+2)$.

REMARK 3. Notice that $u$ is purely imaginary, and $\mathbf{u} \in \mathbb{H}^{n-1}$, following from the fact that $\omega\left(D_{x}\right)$ is in $\mathfrak{s p}(n+2)$.

REMARK 4. Other choices of coframe tend to destroy the scalar-vector character of the analysis and complicate matters tremendously.

Now $\omega\left(D_{t}\right)$ must be a typical element of $\mathfrak{g}$ which we write as follows:

$$
\omega\left(D_{t}\right)=\left(\begin{array}{ccc}
m_{11} & m_{12} & -\overline{\mathbf{m}}_{1}^{t} \\
m_{21} & m_{22} & -\overline{\mathbf{m}}_{2}^{t} \\
\mathbf{m}_{1} & \mathbf{m}_{2} & \mathbf{M}
\end{array}\right) .
$$

Assuming that the curvature form $\Omega$ is zero on the curve flow, the Cartan structure equation evaluated at the evolutionary vector fields $D_{x}, D_{t}$ is as follows:

$$
D_{x} \omega\left(D_{t}\right)-D_{t} \omega\left(D_{x}\right)+\left[\omega\left(D_{t}\right), \omega\left(D_{x}\right)\right]=0 .
$$

Before we explore the Cartan structure equation, let us define some notation. Commutators of vectors and scalars are defined by

$$
C_{\mathbf{u}} \mathbf{m}_{2}:=\left\langle\mathbf{u}, \mathbf{m}_{2}\right\rangle-\left\langle\mathbf{m}_{2}, \mathbf{u}\right\rangle, \quad C_{u} m_{22}:=u m_{22}-m_{22} u
$$

where the inner product $\langle\cdot, \cdot\rangle$ is the Hermitian inner product. Right multiplication by scalar $u$ on vector $\mathbf{h}$ and left multiplication by vector $\mathbf{u}$ on scalar $h$ are defined, respectively, by

$$
R_{u} \mathbf{h}=\mathbf{h} u, \quad L_{\mathbf{u}} h=\mathbf{u} h .
$$

On the other hand, the anti-commutators on vector and scalar quantities are defined by

$$
A_{\mathbf{u}} \mathbf{h}=\langle\mathbf{u}, \mathbf{h}\rangle+\langle\mathbf{h}, \mathbf{u}\rangle, \quad A_{u} h=u h+h u .
$$

Now we explicitly write the components of the Cartan structure equation. Among these equations, the four first equations are concerned with the curvature part in $\mathfrak{h}$ and the last three with the torsion part in $\mathfrak{m}=\mathfrak{g} / \mathfrak{h}$. These equations lead to evolution of the scalar invariant $u$ and the vector invariant $\mathbf{u}$ as combination of geometric operators applied on the proper torsion variables of $\omega\left(D_{t}\right)$ according to the theorem below, in 
which we have defined $\mathfrak{H}_{1}$, similar to that of (4) in Euclidean geometry, as the operator acting on vectors by

$$
\mathfrak{H}_{1} \mathbf{h}=\left(D_{x}^{-1}\left(\mathbf{h}^{t}-\mathbf{u h}^{t}\right)\right) \mathbf{u},
$$

where, for instance, $\mathbf{h} \overline{\mathbf{u}}^{t}$ is the outer product of a vector and a covector, that is, a matrix. Hence, for instance, we can write $\mathbf{M u}=\mathfrak{H}_{1} \mathbf{m}_{2}$ when we see in $(7 \mathrm{c})$ below.

$$
\begin{gathered}
D_{x} m_{11}-m_{12}-m_{21}=0 \\
D_{x} m_{22}-D_{t} u-C_{u} m_{22}+C_{\mathbf{u}} \mathbf{m}_{2}+m_{12}+m_{21}=0 \\
D_{x} \mathbf{m}_{2}-D_{t} \mathbf{u}+R_{u} \mathbf{m}_{2}+\mathfrak{H}_{1} \mathbf{m}_{2}-L_{\mathbf{u}} m_{22}+\mathbf{m}_{1}=0 \\
D_{x} \mathbf{M}-\mathbf{m}_{2} \overline{\mathbf{u}}^{t}+\overline{\mathbf{u m}}_{2}{ }^{t}=0 \\
D_{x} \mathbf{m}_{1}-\mathbf{m}_{2}-\mathbf{u} m_{21}=0 \\
D_{x} m_{12}+m_{11}-m_{22}+m_{12} u-\left\langle\mathbf{m}_{1}, \mathbf{u}\right\rangle=0 \\
D_{x} m_{21}+m_{11}-m_{22}-u m_{21}+\left\langle\mathbf{u}, \mathbf{m}_{1}\right\rangle=0
\end{gathered}
$$

Solving these equations we obtain

THEOREM 1. The evolution of differential invariants can be written in the form

$$
\left(\begin{array}{c}
D_{t} u \\
D_{t} \boldsymbol{u}
\end{array}\right)=\mathfrak{H} \mathfrak{I}\left(\begin{array}{c}
m_{12}+m_{21} \\
\boldsymbol{m}_{1}
\end{array}\right)+\mathfrak{A}\left(\begin{array}{c}
m_{12}+m_{21} \\
\boldsymbol{m}_{1}
\end{array}\right)
$$

where

$$
\mathfrak{H}=\left(\begin{array}{cc}
D_{x}-C_{u} & C_{\boldsymbol{u}} \\
-L_{\boldsymbol{u}} & D_{x}+R_{u}+\mathfrak{H}_{1}
\end{array}\right), \quad \mathfrak{A}=\left(\begin{array}{cc}
\left(2 D_{x}-C_{u}\right) D_{x}^{-1} & 0 \\
-L_{\boldsymbol{u}} D_{x}^{-1} & I
\end{array}\right),
$$

and

$$
\mathfrak{I}=\left(\begin{array}{cc}
\frac{1}{2} D_{x}-\frac{1}{4} C_{u}-\frac{1}{4} A_{u} D_{x}^{-1} \frac{1}{2} A_{u} & \frac{1}{2} C_{\boldsymbol{u}}+\frac{1}{2} u D_{x}^{-1} A_{\boldsymbol{u}} \\
-\frac{1}{2} L_{\boldsymbol{u}} D_{x}^{-1} \frac{1}{2} A_{u}-\frac{1}{2} L_{\boldsymbol{u}} & D_{x}+\frac{1}{2} L_{\boldsymbol{u}} D_{x}^{-1} A_{\boldsymbol{u}}
\end{array}\right)
$$

The proof can be found in [3]. Here we emphasize the following fact. If we subtract (7f) from $(7 \mathrm{~g})$, we deduce that

$$
D_{x}\left(m_{21}-m_{12}\right)=u m_{21}+m_{12} u-A_{\mathbf{u}} \mathbf{m}_{1} .
$$

Using the fact that $\frac{1}{2} A_{u}\left(m_{12}+m_{21}\right)=m_{12} u+u m_{21}$ we obtain that

$$
m_{21}-m_{12}=D_{x}^{-1}\left(\frac{1}{2} A_{u}\left(m_{12}+m_{21}\right)-A_{\mathbf{u}} \mathbf{m}_{1}\right) .
$$

The left-hand side is nothing but the Killing form of two elements of the underlying Lie algebra. Indeed

$$
m_{21}-m_{21}=D_{x}^{-1} K\left(\left(\begin{array}{ccc}
0 & 0 & 0 \\
0 & u & -\overline{\mathbf{u}}^{t} \\
0 & \mathbf{u} & 0
\end{array}\right),\left(\begin{array}{ccc}
0 & 0 & 0 \\
0 & m_{12}+m_{21} & -\overline{\mathbf{m}}_{1}^{t} \\
0 & \mathbf{m}_{1} & 0
\end{array}\right)\right)
$$


Of course up to some constant coefficient. Now let us put $\left(\begin{array}{l}h \\ \mathbf{h}\end{array}\right)=\mathfrak{A}\left(\begin{array}{c}m_{12}+m_{21} \\ \mathbf{m}_{1}\end{array}\right)$. Then we obtain

$$
\left(\begin{array}{c}
m_{12}+m_{21} \\
\mathbf{m}_{1}
\end{array}\right)=\mathfrak{A}^{-1}\left(\begin{array}{l}
h \\
\mathbf{h}
\end{array}\right), \quad \mathfrak{A}^{-1}=\left(\begin{array}{cc}
D_{x}\left(2 D_{x}-C_{u}\right)^{-1} & 0 \\
L_{\mathbf{u}}\left(2 D_{x}-C_{u}\right)^{-1} & I
\end{array}\right) .
$$

Hence the evolution in the theorem takes the following form:

$$
\left(\begin{array}{c}
D_{t} u \\
D_{t} \mathbf{u}
\end{array}\right)=\mathfrak{R}\left(\begin{array}{l}
h \\
\mathbf{h}
\end{array}\right)+\left(\begin{array}{l}
h \\
\mathbf{h}
\end{array}\right), \quad \mathfrak{R}=\mathfrak{H} \mathfrak{I} \mathfrak{A}^{-1}
$$

If we make the specialization $\left(\begin{array}{l}h \\ \mathbf{h}\end{array}\right)=\left(\begin{array}{l}u_{1} \\ \mathbf{u}_{1}\end{array}\right)$, where $u_{1}, \mathbf{u}_{1}$ are the derivatives of $u$ and $\mathbf{u}$ with respect to $x$, respectively, then we obtain the non-commutative evolution equations:

$$
\left\{\begin{array}{l}
u_{t}=\frac{1}{4} u_{3}+\frac{3}{8}\left(-u u_{1} u-u u_{2}+u_{2} u\right)+\frac{3}{4} C_{\mathbf{u}}\left(\mathbf{u} u_{1}\right)+\frac{3}{2} C_{\mathbf{u}}\left(\mathbf{u}_{1} u\right)+\frac{3}{2} C_{\mathbf{u}} \mathbf{u}_{2} \\
\mathbf{u}_{t}=\mathbf{u}_{3}+\frac{3}{2} \mathbf{u}_{2} u+\frac{3}{4} \mathbf{u}_{1}\left(u_{1}+\frac{1}{2} u^{2}+2\langle\mathbf{u}, \mathbf{u}\rangle\right)
\end{array}\right.
$$

The reduction $\mathbf{u}=0$ leads to the second version of the quaternionic non-commutative mKDV scalar equation and the reduction $u=0$ yields the quaternionic vector mKDV equation.

5. Geometric operators expressed in the Killing form. In the method we are using, the only tools we have are the Lie algebra and the Cartan geometry, hence we expect to be able to write the geometric operators $\mathfrak{H}$ and $\mathfrak{I}$ in terms of the Lie bracket, the Killing form and proper projections as we did in the case of Euclidean geometry, see (5).

Let us define the projections $\pi_{0}$ and $\pi_{1}$ as follows:

$$
\pi_{0}\left(\begin{array}{ccc}
m_{11} & 0 & 0 \\
0 & m_{22} & -\overline{\mathbf{m}}_{2}^{t} \\
0 & \mathbf{m}_{2} & \mathbf{M}
\end{array}\right)=\left(\begin{array}{ccc}
0 & 0 & 0 \\
0 & 0 & 0 \\
0 & 0 & \mathbf{M}
\end{array}\right)
$$

and

$$
\pi_{1}\left(\begin{array}{ccc}
m_{11} & 0 & 0 \\
0 & m_{22} & -\overline{\mathbf{m}}_{2}^{t} \\
0 & \mathbf{m}_{2} & \mathbf{M}
\end{array}\right)=\left(\begin{array}{ccc}
0 & 0 & 0 \\
0 & m_{22} & -\overline{\mathbf{m}}_{2}^{t} \\
0 & \mathbf{m}_{2} & 0
\end{array}\right)
$$

Let $\hat{u}$ and $\hat{m}_{2}$ be the projection of $\omega\left(D_{x}\right)$ and $\omega\left(D_{t}\right)$ over the Lie subalgebra $\mathfrak{h}$, respectively, as well as $\hat{a}$ and $\hat{m}_{1}$, the projections of $\omega\left(D_{x}\right)$ and $\omega\left(D_{t}\right)$ over the vector space $\mathfrak{g} / \mathfrak{h}$ which itself is indeed the dual orthogonal of $\mathfrak{h}$ with respect to the Killing form. In other words

$$
\hat{u}=\left(\begin{array}{ccc}
0 & 0 & 0 \\
0 & u & -\overline{\mathbf{u}}^{t} \\
0 & \mathbf{u} & 0
\end{array}\right), \quad \hat{a}=\left(\begin{array}{ccc}
0 & 1 & 0 \\
-1 & 0 & 0 \\
0 & 0 & 0
\end{array}\right)
$$


and

$$
\hat{m}_{2}=\left(\begin{array}{ccc}
m_{11} & 0 & 0 \\
0 & m_{22} & -\overline{\mathbf{m}}_{2}^{t} \\
0 & \mathbf{m}_{2} & \mathbf{M}
\end{array}\right), \quad \hat{m}_{1}=\left(\begin{array}{ccc}
0 & m_{12} & -\overline{\mathbf{m}}_{1}^{t} \\
m_{21} & 0 & 0 \\
\mathbf{m}_{1} & 0 & 0
\end{array}\right) .
$$

In the following lemma, we give the Lie algebraic form of the operator $\mathfrak{H}_{1}$ defined as in (6).

LEMMA 1. Using the setting above, we have that

$$
a d_{\hat{u}} D_{x}^{-1} \pi_{0} a d_{\hat{u}} \pi_{1} \hat{m}_{2}=\left(\begin{array}{ccc}
0 & 0 & 0 \\
0 & 0 & \overline{\mathfrak{H}}_{1} \boldsymbol{m}_{2} \\
0 & -\mathfrak{H}_{1} \boldsymbol{m}_{2} & 0
\end{array}\right) .
$$

Proof. The proof just follows from computing the Lie bracket of the elements of the Lie algebra $\mathfrak{s p}(n+2)$.

Now let us define the projections $\rho_{0}$ and $\rho_{1}$ on the diagonal and off diagonal of the image of $\pi_{1}$ as follows:

$$
\rho_{1}\left(\begin{array}{ccc}
0 & 0 & 0 \\
0 & m_{22} & -\overline{\mathbf{m}}_{2}^{t} \\
0 & \mathbf{m}_{2} & 0
\end{array}\right)=\left(\begin{array}{ccc}
0 & 0 & 0 \\
0 & m_{22} & 0 \\
0 & 0 & 0
\end{array}\right)
$$

and

$$
\rho_{0}\left(\begin{array}{ccc}
0 & 0 & 0 \\
0 & m_{22} & -\overline{\mathbf{m}}_{2}^{t} \\
0 & \mathbf{m}_{2} & 0
\end{array}\right)=\left(\begin{array}{ccc}
0 & 0 & 0 \\
0 & 0 & -\overline{\mathbf{m}}_{2}^{t} \\
0 & \mathbf{m}_{2} & 0
\end{array}\right)
$$

We give some identities which in fact are interactions of projections $\rho_{0}, \rho_{1}$ and $\pi_{1}$.

Proposition 2. For every matrix $\hat{q}$ in the image of $\pi_{1}$, we have that

1. $\left(\frac{1}{2} \rho_{1}+\rho_{0}\right) \pi_{1} a d_{a}^{2} \hat{q}=-\hat{q}$.

2. $\pi_{1} a d_{\hat{a}} a d_{\hat{u}} a d_{\hat{a}} \hat{q}=-a d_{\hat{u}} \rho_{1} \hat{q}-\pi_{1} a d_{\rho_{0} \hat{u}} \rho_{0} \hat{q}$.

The following theorem describes how we can express the geometric operator in terms of Lie algebraic notions, such as Killing form, adjoint representation and the projections.

THEOREM 2. The evolution of the $\hat{u}$ following the Cartan structure equation on $\mathrm{M}=G / H$ can be expressed as

$$
\hat{u}_{t}=\hat{\mathfrak{H}} \hat{\mathfrak{I}} \hat{m}_{0}+\hat{\mathfrak{A}} \hat{m}_{0}, \quad \hat{m}_{0}=\pi_{1} a d_{\hat{a}} \hat{m}_{1} \in \pi_{1} \mathfrak{h},
$$

in which the Lie algebra form $\hat{\mathfrak{I}}$ of geometric operator $\mathfrak{I}$ and $\hat{\mathfrak{A}}$ of the operator $\mathfrak{A}$ appears as

$$
\begin{aligned}
\hat{\mathfrak{H}} & =D_{x}-\pi_{1} a d_{\hat{u}}-a d_{\hat{u}} D_{x}^{-1} \pi_{0} a d_{\hat{u}}, \\
\hat{\mathfrak{I}} & =-\frac{1}{2} \hat{u} D_{x}^{-1} K(\hat{u}, .)-\left(\frac{1}{2} \rho_{1}+\rho_{0}\right) \pi_{1} a d_{\hat{a}}\left(D_{x}-a d_{\hat{u}}\right) a d_{\hat{a}} \pi_{1}\left(\frac{1}{2} \rho_{1}+\rho_{0}\right), \\
\hat{\mathfrak{A}} & =\rho_{0}+2 \rho_{1}-a d_{\hat{u}} D_{x}^{-1} \rho_{1} .
\end{aligned}
$$


Proof. From the curvature part or in fact the equations (7b), (7c) and (7d) and the previous lemma, we simply find that

$$
\hat{u}_{t}=\hat{\mathfrak{H}}\left(\pi_{1} \hat{m}_{2}\right)+\hat{m}_{0}, \quad \hat{m}_{0}=\pi_{1} \operatorname{ad}_{\hat{a}} \hat{m}_{1}
$$

now the torsion part gives the following matrix equation:

$$
\operatorname{ad}_{\hat{a}}\left(\hat{m}_{2}\right)=\left(D_{x}-\operatorname{ad}_{\hat{u}}\right) \hat{m}_{1} .
$$

Since

$$
\operatorname{ad}_{\hat{a}}^{2} \neq \lambda I, \quad \lambda \in \mathbb{R}
$$

we cannot solve equation (13) in the usual way. Therefore the existence of the Nijenhuis operator $\mathfrak{A}$ plays a crucial rule in the symplectic geometry. Notice that in the Riemannian case we do have $\operatorname{ad}_{\hat{a}}^{2}=-I$.

In order to get rid of this difficulty, we do as follows:

$$
\begin{aligned}
\hat{u}_{t} & =\hat{\mathfrak{H}}\left(\begin{array}{ccc}
0 & 0 & 0 \\
0 & m_{22}-m_{11} & -\overline{\mathbf{m}}_{2}^{t} \\
0 & \mathbf{m}_{2} & 0
\end{array}\right)+\hat{\mathfrak{H}}\left(\begin{array}{ccc}
0 & 0 & 0 \\
0 & m_{11} & 0 \\
0 & 0 & 0
\end{array}\right)+\hat{m}_{0} \\
& =\left(\begin{array}{ccc}
0 & 0 & 0 \\
0 & m_{22}-m_{11} & -\overline{\mathbf{m}}_{2}^{t} \\
0 & \mathbf{m}_{2} & 0
\end{array}\right)+\left(D_{x}-\pi_{1} a d_{\hat{u}}\right)\left(\begin{array}{ccc}
0 & 0 & 0 \\
0 & m_{11} & 0 \\
0 & 0 & 0
\end{array}\right)+\hat{m}_{0} \\
& =\hat{\mathfrak{H}}\left(\begin{array}{ccc}
0 & 0 & 0 \\
0 & m_{22}-m_{11} & -\overline{\mathbf{m}}_{2}^{t} \\
0 & \mathbf{m}_{2} & 0
\end{array}\right)+\left(\left(2 D_{x}-\mathrm{ad}_{\hat{u}}\right) D_{x}^{-1} \rho_{1}+\rho_{0}\right) \hat{m}_{0} \\
& =\hat{\mathfrak{H}}\left(\begin{array}{ccc}
0 & 0 & 0 \\
0 & m_{22}-m_{11} & -\overline{\mathbf{m}}_{2}^{t} \\
0 & \mathbf{m}_{2} & 0
\end{array}\right)+\hat{\mathfrak{A}}\left(\hat{m}_{0} .\right.
\end{aligned}
$$

Now the matrix

$$
\left(\begin{array}{ccc}
0 & 0 & 0 \\
0 & m_{22}-m_{11} & -\overline{\mathbf{m}}_{2}^{t} \\
0 & \mathbf{m}_{2} & 0
\end{array}\right)
$$

can be expressed in terms of $\hat{m}_{2}$ and consequently in terms of $\hat{m}_{1}$ using the identity (13) as follows:

$$
\begin{aligned}
\left(\begin{array}{ccc}
0 & 0 & 0 \\
0 & m_{22}-m_{11} & -\overline{\mathbf{m}}_{2}^{t} \\
0 & \mathbf{m}_{2} & 0
\end{array}\right) & =-\left(\frac{1}{2} \rho_{1}+\rho_{0}\right) \pi_{1} \mathrm{ad}_{a}^{2} \hat{m}_{2} \\
& =-\left(\frac{1}{2} \rho_{1}+\rho_{0}\right) \pi_{1} \operatorname{ad}_{a}\left(D_{x}-\operatorname{ad}_{\hat{u}}\right) \hat{m}_{1}
\end{aligned}
$$


According to identity (9) we have that $m_{21}-m_{12}=D_{x}^{-1} K\left(\hat{u}, \hat{m}_{0}\right)$, thus the matrix $\hat{m}_{1}$ can be written in terms of $\hat{m}_{0}$ as follows.

$$
\begin{aligned}
\hat{m}_{1}= & \left(\begin{array}{ccc}
0 & \frac{m_{12}-m_{21}}{2} & 0 \\
\frac{m_{21}-m_{12}}{2} & 0 & 0 \\
0 & 0 & 0
\end{array}\right) \\
& +\left(\begin{array}{ccc}
0 & \frac{m_{12}+m_{21}}{2} & -\overline{\mathbf{m}}_{1}^{t} \\
\frac{m_{21}+m_{12}}{2} & 0 & 0 \\
\mathbf{m}_{1} & 0 & 0
\end{array}\right) \\
= & -\frac{1}{2} \hat{a} D_{x}^{-1} K\left(\hat{u}, \hat{m}_{0}\right)+\operatorname{ad}_{\hat{a}}\left(\frac{1}{2} \rho_{1}+\rho_{0}\right) \hat{m}_{0} .
\end{aligned}
$$

Hence we identify the operator $\hat{\mathfrak{I}}$ as follows:

$$
\begin{aligned}
&\left(\begin{array}{ccc}
0 & 0 & 0 \\
0 & m_{22}-m_{11} & -\overline{\mathbf{m}}_{2}^{t} \\
0 & \mathbf{m}_{2} & 0
\end{array}\right) \\
&=-\left(\frac{1}{2} \rho_{1}+\rho_{0}\right) \pi_{1} \operatorname{ad}_{\hat{a}}\left(D_{x}-\operatorname{ad}_{\hat{u}}\right) \hat{m}_{1} \\
&=-\left(\frac{1}{2} \rho_{1}+\rho_{0}\right) \pi_{1} \operatorname{ad}_{\hat{a}}\left(D_{x}-\operatorname{ad}_{\hat{u}}\right)\left(-\frac{1}{2} \hat{a} D_{x}^{-1} K\left(\hat{u}, \hat{m}_{0}\right)+\operatorname{ad}_{\hat{a}}\left(\frac{1}{2} \rho_{1}+\rho_{0}\right) \hat{m}_{0}\right) \\
&=-\frac{1}{2}\left(\frac{1}{2} \rho_{1}+\rho_{0}\right) \pi_{1} \operatorname{ad}_{\hat{a}}\left(\operatorname{ad}_{\hat{u}} \hat{a}\right) D_{x}^{-1} K\left(\hat{u}, \hat{m}_{0}\right) \\
&-\left(\frac{1}{2} \rho_{1}+\rho_{0}\right) \pi_{1} \operatorname{ad}_{\hat{a}}\left(D_{x}-\operatorname{ad}_{\hat{u}}\right) \operatorname{ad}_{\hat{a}}\left(\frac{1}{2} \rho_{1}+\rho_{0}\right) \hat{m}_{0} \\
&=+\frac{1}{2}\left(\frac{1}{2} \rho_{1}+\rho_{0}\right) \pi_{1}\left(\operatorname{ad}_{\hat{a}}^{2} \hat{u}\right) D_{x}^{-1} K\left(\hat{u}, \hat{m}_{0}\right) \\
&-\left(\frac{1}{2} \rho_{1}+\rho_{0}\right) \pi_{1} \operatorname{ad}_{\hat{a}}\left(D_{x}-\operatorname{ad}_{\hat{u}}\right) a d_{\hat{a}}\left(\frac{1}{2} \rho_{1}+\rho_{0}\right) \hat{m}_{0} \\
&=-\frac{1}{2} \hat{u} D_{x}^{-1} K\left(\hat{u}, \hat{m}_{0}\right)-\left(\frac{1}{2} \rho_{1}+\rho_{0}\right) \pi_{1} a d_{\hat{a}}\left(D_{x}-\operatorname{ad}_{\hat{u}}\right) \operatorname{ad}_{\hat{a}}\left(\frac{1}{2} \rho_{1}+\rho_{0}\right) \hat{m}_{0} \\
&=-\frac{1}{2} \hat{u} D_{x}^{-1} K\left(\hat{u}, \hat{m}_{0}\right)-\left(\frac{1}{2} \rho_{1}+\rho_{0}\right) \pi_{1} a d_{\hat{a}}\left(D_{x}-\operatorname{ad}_{\hat{u}}\right) \operatorname{ad}_{\hat{a}} \pi_{1}\left(\frac{1}{2} \rho_{1}+\rho_{0}\right) \hat{m}_{0} \\
&= \hat{\mathfrak{I}} .
\end{aligned}
$$

In the last line, we add $\pi_{1}$ at end to have a symmetrical expression, as it does not change anything. Thus replacing the last equation into the evolution (14), we obtain

$$
\hat{u}_{t}=\hat{\mathfrak{H}} \hat{\mathfrak{I}} \hat{m}_{0}+\hat{\mathfrak{A}} \hat{m}_{0}
$$


Remark 5. This is exactly the Poisson operator in $[\mathbf{1 7}, 1.13]$ which in general is defined on Hermitian symmetric spaces. See also $[\mathbf{1}, \mathbf{1 6}, \mathbf{1 8}]$.

6. Geometric operator. As a main result of the paper, it can be shown that the operators appeared so far will construct the Hamiltonian and symplectic operators as well as a Nijenhuis operator. The basic theory of Hamiltonian, symplectic and Nijenhuis operators for scalar evolution equations is given in $[\mathbf{5}, \mathbf{1 2}]$. The presentation here will apply this theory to a Lie algebra valued setting. In the definition below, $\pi_{1} \mathfrak{h}$ is denoted to the space of all $\pi_{1} \mathfrak{h}$-valued functions defined on $x$-jet space $\left(x, \hat{u}, \hat{u}_{1}, \ldots\right)=$ $J^{\infty}$ of the dynamical variable $\hat{u}$ and its derivatives. This space is a Lie algebra if we consider its elements as vector field $\hat{p} \cdot \frac{\partial}{\partial \hat{u}}$ where $\hat{p} \in \pi_{1} \mathfrak{h}$.

Definition 7. The linear operator $N: \pi_{1} \mathfrak{h} \rightarrow \pi_{1} \mathfrak{h}$ is a Nijenhuis operator if and only if

$$
D_{N}[N \hat{q}](\hat{p})-D_{N}[N \hat{p}](\hat{q})+N\left(D_{N}[\hat{p}](\hat{q})-D_{N}[\hat{q}](\hat{p})\right)=0,
$$

for any pair of vector fields $\hat{p}, \hat{q} \in \pi_{1} \mathfrak{h}$. Here $D_{N}$ denotes the Fréchet derivative of $N$ is defined as a Linear map by

$$
D_{N}[\hat{q}]=\left.\frac{d}{d \epsilon} N[\hat{u}+\epsilon \hat{q}]\right|_{\epsilon=0} .
$$

The defining relation for this operator was originally found as a necessary condition for an almost complex structure to be complex, i.e., as an integrability condition. It is used to construct hierarchies of integrable equations.

THEOREM 3. The operator $N=\hat{\mathfrak{A}}$ is indeed a Nijenhuis operator. That is, the Nijenhuis tensor vanishes.

Proof. Here we are given

$$
N \hat{p}=\left(\rho_{0}+2 \rho_{1}\right) \hat{p}-\operatorname{ad}_{\hat{u}} D_{x}^{-1} \rho_{1} \hat{p} .
$$

We compute the Fréchet derivative of $N$ as follows:

$$
D_{N}[\hat{q}](\hat{p})=-\operatorname{ad}_{\hat{q}} D_{x}^{-1} \rho_{1} \hat{p} .
$$

Now we see that two first terms of the right-hand side of (16) can be derived as below.

$$
\begin{aligned}
D_{N}[N \hat{q}](\hat{p})-D_{N}[N \hat{p}](\hat{q})= & -\operatorname{ad}_{N \hat{q}} D_{x}^{-1} \rho_{1} \hat{p}+\operatorname{ad}_{\hat{p}} D_{x}^{-1} \rho_{1} \hat{q} \\
= & -\operatorname{ad}_{\left(\rho_{0}+2 \rho_{1}\right) \hat{q}-\operatorname{ad}_{\hat{u}} D_{x}^{-1} \rho_{1} \hat{q}} D_{x}^{-1} \rho_{1} \hat{p}+\operatorname{ad}_{\left(\rho_{0}+2 \rho_{1}\right) \hat{p}-\operatorname{ad}_{\hat{u}} D_{x}^{-1} \rho_{1} \hat{p}} D_{x}^{-1} \rho_{1} \hat{q} \\
= & -\operatorname{ad}_{\hat{q}} D_{x}^{-1} \rho_{1} \hat{p}-\operatorname{ad}_{\rho_{1} \hat{q}} D_{x}^{-1} \rho_{1} \hat{p}+\operatorname{ad}_{\operatorname{ad}_{\hat{u}} D_{x}^{-1} \rho_{1} \hat{q}} D_{x}^{-1} \rho_{1} \hat{p} \\
& +\operatorname{ad}_{\hat{p}} D_{x}^{-1} \rho_{1} \hat{q}+\operatorname{ad}_{\rho_{1} \hat{p}} D_{x}^{-1} \rho_{1} \hat{q}-\operatorname{ad}_{\operatorname{ad}_{\hat{u}} D_{x}^{-1} \rho_{1} \hat{p}} D_{x}^{-1} \rho_{1} \hat{q} .
\end{aligned}
$$

On the other hand, for the two last terms of the right-hand side of (16) we obtain

$$
\begin{aligned}
N\left(D_{N}[\hat{p}](\hat{q})-D_{N}[\hat{q}](\hat{p})\right)= & \left(\rho_{0}+2 \rho_{1}\right)\left(-\operatorname{ad}_{\hat{p}} D_{x}^{-1} \rho_{1} \hat{q}+\operatorname{ad}_{\hat{q}} D_{x}^{-1} \rho_{1} \hat{p}\right) \\
& -\operatorname{ad}_{\hat{u}} D_{x}^{-1} \rho_{1}\left(-\operatorname{ad}_{\hat{p}} D_{x}^{-1} \rho_{1} \hat{q}+\operatorname{ad}_{\hat{q}} D_{x}^{-1} \rho_{1} \hat{p}\right) \\
= & -\operatorname{ad}_{\hat{p}} D_{x}^{-1} \rho_{1} \hat{q}-\operatorname{ad}_{\rho_{1} \hat{p}} D_{x}^{-1} \rho_{1} \hat{q}+\operatorname{ad}_{\hat{q}} D_{x}^{-1} \rho_{1} \hat{p}+\operatorname{ad}_{\rho_{1} \hat{q}} D_{x}^{-1} \rho_{1} \hat{p} \\
& +\operatorname{ad}_{\hat{u}} D_{x}^{-1} \operatorname{ad}_{\rho_{1} \hat{p}} D_{x}^{-1} \rho_{1} \hat{q}-\operatorname{ad}_{\hat{u}} D_{x}^{-1} \operatorname{ad}_{\rho_{1} \hat{q}} D_{x}^{-1} \rho_{1} \hat{p} .
\end{aligned}
$$


Now we replace these equations into the right-hand side of (16) and then simplify it as follows, using the Jacobi identity, in order to prove the statement of the theorem.

$$
\begin{aligned}
D_{N}[ & N \hat{q}](\hat{p})-D_{N}[N \hat{p}](\hat{q})+N\left(D_{N}[\hat{p}](\hat{q})-D_{N}[\hat{q}](\hat{p})\right) \\
= & +\operatorname{ad}_{\operatorname{ad}_{\hat{u}} D_{x}^{-1} \rho_{1} \hat{q}} D_{x}^{-1} \rho_{1} \hat{p}-\operatorname{ad}_{\mathrm{ad}_{\hat{u}} D_{x}^{-1} \rho_{1} \hat{p}} D_{x}^{-1} \rho_{1} \hat{q} \\
& +\operatorname{ad}_{\hat{u}} D_{x}^{-1} \operatorname{ad}_{\rho_{1} \hat{p}} D_{x}^{-1} \rho_{1} \hat{q}-\operatorname{ad}_{\hat{u}} D_{x}^{-1} \operatorname{ad}_{\rho_{1} \hat{q}} D_{x}^{-1} \rho_{1} \hat{p} \\
= & -\operatorname{ad}_{\hat{u}} \operatorname{ad}_{D_{x}^{-1} \rho_{1} \hat{p}} D_{x}^{-1} \rho_{1} \hat{q} \\
& +\operatorname{ad}_{\hat{u}} D_{x}^{-1} \operatorname{ad}_{\rho_{1} \hat{p}} D_{x}^{-1} \rho_{1} \hat{q}-\operatorname{ad}_{\hat{u}} D_{x}^{-1} \operatorname{ad}_{\rho_{1} \hat{q}} D_{x}^{-1} \rho_{1} \hat{p} \\
= & -\operatorname{ad}_{\hat{u}} D_{x}^{-1} \operatorname{ad}_{\rho_{1} \hat{p}} D_{x}^{-1} \rho_{1} \hat{q}+\operatorname{ad}_{\hat{u}} D_{x}^{-1} \operatorname{ad}_{\rho_{1} \hat{q}} D_{x}^{-1} \rho_{1} \hat{p} \\
& +\operatorname{ad}_{\hat{u}} D_{x}^{-1} \operatorname{ad}_{\rho_{1} \hat{p}} D_{x}^{-1} \rho_{1} \hat{q}-\operatorname{ad}_{\hat{u}} D_{x}^{-1} \operatorname{ad}_{\rho_{1} \hat{q}} D_{x}^{-1} \rho_{1} \hat{p} \\
= & 0 .
\end{aligned}
$$

REMARK 6. Notice that operator $\hat{\mathfrak{A}}$ is not a recursion operator, that is, it is not invariant under the evolutionary flow.

REMARK 7. Since $N=\hat{\mathfrak{A}}$ is invertible, so by [5, proposition 3.2], the operator $\hat{\mathfrak{A}}^{-1}$ itself is Nijenhuis operator.

Now let $\hat{p}$ be an element of $\pi_{1} \mathfrak{h}_{1}$. Then the covector field $\hat{p}$.d $\hat{u}$ is dual to the vector field $\hat{q} \cdot \frac{\partial}{\partial \hat{u}}$ defined by the following pairing in our context

$$
\left\langle\hat{p} \cdot d \hat{u}, \hat{q} \cdot \frac{\partial}{\partial \hat{u}}\right\rangle=\int K(\hat{p}, \hat{q}) .
$$

We shortly write this pairing as $\langle\hat{p}, \hat{q}\rangle$ between the vector field $\hat{q} \cdot \frac{\partial}{\partial \hat{u}}$ identified with $\hat{q}$ and covector field $\hat{p}$.d $\hat{u}$ identified with $\hat{p}$.

The adjoint of the operator $P$ is defined as

$$
\langle\hat{p}, P \hat{q}\rangle=\left\langle P^{*} \hat{p}, \hat{q}\right\rangle .
$$

Since the pairing is non-degenerate, $P^{*}$ is well-defined. This definition can be extend to the operators acting on the vector fields or covector fields. Using the definition above one can simply prove the following lemma.

Lemma 2. The adjoint of the Nijenhuis operator $\hat{\mathfrak{A}}$ is $\hat{\mathfrak{A}}^{*}=\rho_{0}+2 \rho_{1}-\rho_{1} D_{x}^{-1} a d_{\hat{u}}$. Furthermore the operators $\hat{\mathfrak{H}}$ and $\hat{\mathfrak{I}}$ are anti-symmetric. Moreover, there is a meaningful link between the operator $\hat{\mathfrak{H}}$ and $\hat{\mathfrak{A}}$, that is,

$$
\hat{\mathfrak{A}} \hat{\mathfrak{H}}=\hat{\mathfrak{H}} \hat{\mathfrak{A}}^{*} .
$$

That means that the operator $\hat{\mathfrak{H}} \hat{\mathfrak{A}}^{*}$ is also antisymmetric.

In the light of Theorem 3 and the lemmas above the Lie algebraic form of the operator $\mathfrak{R}$ defined in (10) can be written as

$$
\hat{\mathfrak{R}}=\hat{\mathfrak{H}} \hat{\mathfrak{I}} \hat{\mathfrak{A}}^{-1}=\left(\hat{\mathfrak{H}} \hat{\mathfrak{A}}^{*}\right)\left(\hat{\mathfrak{A}}^{-1 *} \hat{\mathfrak{I}} \hat{\mathfrak{A}}^{-1}\right) .
$$


This decomposition of the operator $\hat{\mathfrak{R}}$ is the key to find Hamiltonian and symplectic operator.

THEOREM 4. The operators $\hat{\mathfrak{H}}$ and $\hat{\mathfrak{H}} \hat{\mathfrak{A}}^{*}$ are Hamiltonian operators and the operator

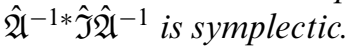

Proof of this theorem is partly presented in the next subsection.

REMARK 8. Theorems 3 and 4 show that the manifold $\mathrm{M}=G / H$ is endowed with a so called 'Poisson-Nijenhuis structure' as is defined in [9]. For more information, the reader is referred to the works of Magri, as presented in for instance [8] and [10].

6.1. Hamiltonian operator. The linear operator $\hat{\mathfrak{H}}$ defined on $\pi_{1} \mathfrak{h}$ is Hamiltonian operator if the Schouten bracket $[\hat{\mathfrak{H}}, \hat{\mathfrak{H}}]$ vanishes. In the case at hand, this bracket can be expressed as follows.

$$
[\hat{\mathfrak{H}}, \hat{\mathfrak{H}}]=\sum_{i=1}^{3} \int K\left(\hat{p}_{i}, D_{\hat{\mathfrak{H}}}\left[\hat{\mathfrak{H}} \hat{p}_{i+2}\right]\left(\hat{p}_{i+1}\right)\right)
$$

NotATION 1. To have more space, we will remove the sum over index $i$, but we take into account the rule of shifting, that is for instance, we can add to index $i$, by 1, 2 and use, for instance, the fact that

$$
\hat{p}_{i+3}=\hat{p}_{i}, \quad \hat{p}_{i+4}=\hat{p}_{i+1},
$$

and so on. Moreover, we simply use the notation $H$ for the operator $\hat{\mathfrak{H}}$ and $p_{i}$ for the matrix $\hat{p}_{i}$ and likewise for $\hat{u}$ so that we write as $H=D_{x}-\pi_{1} \operatorname{ad}_{u}-\operatorname{ad}_{u} D_{x}^{-1} \pi_{0} \operatorname{ad}_{u}$.

The operator $H$ can be expressed as a combination of three simpler operators as

$$
H=H_{1}+H_{2}+H_{3}, \quad H_{1}=-D_{x}, H_{2}=-\pi_{1} \operatorname{ad}_{u}, \quad H_{3}=-\operatorname{ad}_{u} D_{x}^{-1} \pi_{0} \operatorname{ad}_{u} .
$$

In order to prove that $H$ is Hamiltonian, we show that $\left[H_{i}, H_{j}\right]=0$, for $i, j=1,2,3$.

LEMmA 3. The Schouten bracket $\left[\mathrm{H}_{3}, \mathrm{H}_{3}\right]$ vanishes.

Proof. We explain every single step and every single rule we use, so that later on we will just do it. By computing the Fréchet derivative, we obtain

$$
\begin{aligned}
{\left[H_{3}, H_{3}\right]\left(p_{1}, p_{2}, p_{3}\right)=} & \int K\left(p_{i}, \operatorname{ad}_{\mathrm{ad}_{u} D_{x}^{-1} \pi_{0} \operatorname{ad}_{u} p_{i+2}} D_{x}^{-1} \pi_{0} \operatorname{ad}_{u} p_{i+1}\right) \\
& +\int K\left(p_{i}, \operatorname{ad}_{u} D_{x}^{-1} \pi_{0} \operatorname{ad}_{\mathrm{ad}_{u} D_{x}^{-1} \pi_{0} \operatorname{ad}_{u} p_{i+2}} p_{i+1}\right) .
\end{aligned}
$$

The first term becomes as follows using the shifting rule (19).

$$
\int K\left(p_{i}, \operatorname{ad}_{\mathrm{ad}_{u} D_{x}^{-1} \pi_{0} \operatorname{ad}_{u} p_{i+2}} D_{x}^{-1} \pi_{0} \operatorname{ad}_{u} p_{i+1}\right)=\int K\left(p_{i+1}, \operatorname{ad}_{\operatorname{ad}_{u} D_{x}^{-1} \pi_{0} \operatorname{ad}_{u} p_{i}} D_{x}^{-1} \pi_{0} \operatorname{ad}_{u} p_{i+2}\right) .
$$

As we know, the Killing form is invariant under adjoint action,

$$
K\left(\operatorname{ad}_{X} Y, Z\right)+K\left(Y, \operatorname{ad}_{X} Z\right)=0, \quad X, Y, X \in \mathfrak{g} .
$$


Now the second term is simplified as below using this rule and integration by parts.

$$
\begin{aligned}
& -\int K\left(\operatorname{ad}_{u} p_{i}, D_{x}^{-1} \pi_{0} \operatorname{ad}_{\mathrm{ad}_{u} D_{x}^{-1} \pi_{0} \mathrm{ad}_{u} p_{i+2}} p_{i+1}\right) \\
& =\int K\left(D_{x}^{-1} \operatorname{ad}_{u} p_{i}, \pi_{0} \operatorname{ad}_{\mathrm{ad}_{u} D_{x}^{-1} \pi_{0} \mathrm{ad}_{u} p_{i+2}} p_{i+1}\right) \\
& =-\int K\left(\operatorname{ad}_{\mathrm{ad}_{u} D_{x}^{-1} \pi_{0} \mathrm{ad}_{u} p_{i+2}} D_{x}^{-1} \pi_{0} \mathrm{ad}_{u} p_{i}, p_{i+1}\right) .
\end{aligned}
$$

Now using the Jacobi identity for the three elements $u, D^{-1} \pi_{0} \operatorname{ad}_{u} p_{i+2}$ and $D^{-1} \pi_{0} \operatorname{ad}_{u} p_{i}$ of the Lie algebra $\mathfrak{g}$, we obtain

$$
\begin{aligned}
{\left[H_{3}, H_{3}\right]\left(p_{1}, p_{2}, p_{3}\right) } & =\int K\left(p_{i+1}, \operatorname{ad}_{u} \operatorname{ad}_{D_{x}^{-1} \pi_{0} \operatorname{ad}_{u} p_{i}} D^{-1} \pi_{0} \operatorname{ad}_{u} p_{i+2}\right) \\
& =-\int K\left(\operatorname{ad}_{u} p_{i+1}, \operatorname{ad}_{D_{x}^{-1} \pi_{0} \operatorname{ad}_{u} p_{i}} D^{-1} \pi_{0} \operatorname{ad}_{u} p_{i+2}\right) \\
& =-\int K\left(\pi_{0} \operatorname{ad}_{u} p_{i+1}, \operatorname{ad}_{D_{x}^{-1} \pi_{0} \operatorname{ad}_{u} p_{i}} D_{x}^{-1} \pi_{0} \operatorname{ad}_{u} p_{i+2}\right) \\
& =-\frac{1}{3} \int D_{x} K\left(D_{x}^{-1} \pi_{0} \operatorname{ad}_{u} p_{i+1}, \operatorname{ad}_{D_{x}^{-1} \pi_{0} \operatorname{ad}_{u} p_{i}} D_{x}^{-1} \pi_{0} \operatorname{ad}_{u} p_{i+2}\right) \\
& =0 .
\end{aligned}
$$

LEMMA 4. $\left[H_{3}, H_{2}\right]=0$.

Proof. The Schouten bracket $\left[H_{3}, H_{2}\right]$ is

$$
\begin{aligned}
{\left[H_{3}, H_{2}\right]\left(p_{1}, p_{2}, p_{3}\right)=} & +\int K\left(p_{i}, \pi_{1} \operatorname{ad}_{\mathrm{ad}_{u} D_{x}^{-1} \pi_{0} \operatorname{ad}_{u} p_{i+2}} p_{i+1}\right) \\
& +\int K\left(p_{i}, \operatorname{ad}_{\pi_{1} \mathrm{ad}_{u} p_{i+2}} D_{x}^{-1} \pi_{0} \mathrm{ad}_{u} p_{i+1}\right) \\
& +\int K\left(p_{i}, \operatorname{ad}_{u} D_{x}^{-1} \pi_{0} \operatorname{ad}_{\pi_{1} \operatorname{ad}_{u} p_{i+2}} p_{i+1}\right) .
\end{aligned}
$$

The first term on the right is simplified as follows:

$$
\begin{aligned}
\int K\left(p_{i}, \pi_{1} \operatorname{ad}_{\left.\operatorname{ad}_{u} D_{x}^{-1} \pi_{0} \operatorname{ad}_{u} p_{i+2} p_{i+1}\right)}\right. & =-\int K\left(\operatorname{ad}_{u} D_{x}^{-1} \pi_{0} \operatorname{ad}_{u} p_{i+2}, \operatorname{ad}_{p_{i}} p_{i+1}\right) \\
& =\int K\left(D_{x}^{-1} \pi_{0} \operatorname{ad}_{u} p_{i+2}, \pi_{0} \operatorname{ad}_{u} \operatorname{ad}_{p_{i}} p_{i+1}\right)
\end{aligned}
$$

The second and third terms of (21) are simplified according to the invariance property of the Killing form (20), Jacobi identity and the fact that

$$
\pi_{0} \operatorname{ad}_{\pi_{1} \operatorname{ad}_{u} p_{i+2}} p_{i}=\pi_{0} \operatorname{ad}_{\mathrm{ad}_{u} p_{i+2}} p_{i},
$$


to get the following expression:

$$
\begin{aligned}
- & \int K\left(\pi_{0} \operatorname{ad}_{\pi_{1} \mathrm{ad}_{u} p_{i+2}} p_{i}, D_{x}^{-1} \pi_{0} \operatorname{ad}_{u} p_{i+1}\right)+K\left(D_{x}^{-1} \pi_{0} \operatorname{ad}_{u} p_{i+1}, \pi_{0} \operatorname{ad}_{\pi_{1} \mathrm{ad}_{u} p_{i}} p_{i+2}\right) \\
= & -\int K\left(\pi_{0} \operatorname{ad}_{\mathrm{ad}_{u} p_{i+2}} p_{i}, D_{x}^{-1} \pi_{0} \mathrm{ad}_{u} p_{i+1}\right)+K\left(D_{x}^{-1} \pi_{0} \mathrm{ad}_{u} p_{i+1}, \pi_{0} \operatorname{ad}_{\mathrm{ad}_{u} p_{i}} p_{i+2}\right) \\
& =\int K\left(\pi_{0} \operatorname{ad}_{u} \operatorname{ad}_{p_{i}} p_{i+2}, D_{x}^{-1} \pi_{0} \mathrm{ad}_{u} p_{i+1}\right) \\
& =\int K\left(\pi_{0} \operatorname{ad}_{u} \operatorname{ad}_{p_{i+1}} p_{i}, D_{x}^{-1} \pi_{0} \operatorname{ad}_{u} p_{i+2}\right) .
\end{aligned}
$$

Now it should be clear that $\left[H_{3}, H_{2}\right]\left(p_{1}, p_{2}, p_{3}\right)$ vanishes.

Lemma 5. $\left[H_{2}, H_{2}\right]=0=\left[H_{1}, H_{2}\right]$.

Proof. We see that $\int K\left(\operatorname{ad}_{p_{i+1}} p_{i}, \operatorname{ad}_{p_{i+2}} u\right)=0$ holds using the Jacobi identity and (20). Also one has $\pi_{0} \operatorname{ad}_{p_{i+1}} p_{i}=\operatorname{ad}_{\rho_{0} p_{i+1}} \rho_{0} p_{i}$. Hence after few simple step, one can find out that

$$
\begin{aligned}
{\left[H_{2}, H_{2}\right]\left(p_{1}, p_{2}, p_{3}\right) } & =\int K\left(p_{i}, \pi_{1} \operatorname{ad}_{\pi_{1} \operatorname{ad}_{u} p_{i+2}} p_{i+1}\right) \\
& =-\int K\left(\operatorname{ad}_{p_{i+1}} p_{i}, \operatorname{ad}_{p_{i+2}} u-\pi_{0} \operatorname{ad}_{p_{i+2}} u\right)=0 .
\end{aligned}
$$

Similarly we have that

$$
\left[H_{1}, H_{2}\right]\left(p_{1}, p_{2}, p_{3}\right)=-\int K\left(p_{i}, \operatorname{ad}_{p_{i+2, x}} p_{i+1}\right)=-\frac{1}{3} \int D_{x} K\left(p_{i}, \operatorname{ad}_{p_{i+2}} p_{i+2}\right)=0 .
$$

This concludes the proof of the lemma.

LEMMA 6. $\left[H_{1}, H_{3}\right]=0$.

Proof. Using the Jacobi identity and (20), one can get that

$$
\begin{aligned}
{\left[H_{1}, H_{3}\right]\left(p_{1}, p_{2}, p_{3}\right) } & =-\int K\left(p_{i}, \operatorname{ad}_{p_{i+2, x}} D_{x}^{-1} \pi_{0} \operatorname{ad}_{u} p_{i+1}\right)-\int K\left(p_{i}, \operatorname{ad}_{u} D_{x}^{-1} \pi_{0} \operatorname{ad}_{p_{i+2, x}} p_{i+1}\right) \\
& =\int K\left(D_{x}^{-1} \operatorname{ad}_{p_{i}} p_{i+2, x}, \pi_{0} \operatorname{ad}_{u} p_{i+1}\right)+\int K\left(\pi_{0} \operatorname{ad}_{u} p_{i+1}, D_{x}^{-1} \operatorname{ad}_{p_{i, x}} p_{i+2}\right) \\
& =\int K\left(\pi_{0} \operatorname{ad}_{u} p_{i+1}, \operatorname{ad}_{p_{i}} p_{i+2}\right) \\
& =\int K\left(\rho_{0} u, \operatorname{ad}_{\rho_{0} p_{i+1}} \operatorname{ad}_{\rho_{0} p_{i}} \rho_{0} p_{i+2}\right)=0 .
\end{aligned}
$$

Now we obtain the main result out of these lemmas that the operator $H$ is a Hamiltonian operator. We can simply prove that the operator $\hat{\mathfrak{H}} \hat{\mathfrak{A}}^{*}$ is also Hamiltonian. In fact we have that $\hat{\mathfrak{H}} \hat{\mathfrak{A}}^{*}=C_{1}+C_{2}$ in which

$$
C_{1}=D_{x}-\pi_{1} \mathrm{ad}_{u}-\mathrm{ad}_{u} D_{x}^{-1} \pi_{0} \mathrm{ad}_{u},
$$


and

$$
C_{2}=D_{x} \rho_{1}-\pi_{1} \operatorname{ad}_{u} \rho_{1}+\rho_{1} \pi_{1} \operatorname{ad}_{u}+\operatorname{ad}_{u} D_{x}^{-1} \rho_{1} \pi_{1} \operatorname{ad}_{u}
$$

We already proved that $C_{1}$ is Hamiltonian, that is, $\left[C_{1}, C_{1}\right]=0$. Similarly we can prove that $\left[C_{2}, C_{2}\right]=0$. It is not difficult to show that $\left[C_{1}, C_{2}\right]=0$.

6.2. Symplectic operator. As is stated in Theorem (4), it is claimed that the operator $S=\hat{\mathfrak{A}}^{-1 *} \hat{\mathfrak{I}} \hat{\mathfrak{A}}^{-1}$ is symplectic. The operator $\hat{\mathfrak{I}}$ can be expressed as combination of three basic operators:

$$
\hat{\mathfrak{I}}=S_{0}+S_{1}+S_{2}
$$

in which

$$
S_{0}=-\frac{1}{2} u D_{x}^{-1} K(u, \cdot), \quad S_{1}=\frac{1}{2} D_{x} \rho_{1}-\frac{1}{4} \operatorname{ad}_{\rho_{1} u} \rho_{1},
$$

and

$$
S_{2}=D_{x} \rho_{0}-\frac{1}{2} \operatorname{ad}_{\rho_{0} u} \rho_{1}-\frac{1}{2} \pi_{1} \operatorname{ad}_{\rho_{0} u} \rho_{0}
$$

In order to prove that the operator $S$ is symplectic, we need to show that the Schouten bracket of the operator vanishes. The local version of Schouten bracket for the linear operator $S$ defined on vector filed $\pi_{1} \mathfrak{h}$ with the covector field valued can be formulated as follows.

$$
[S, S]=\sum_{i=1}^{3} \int K\left(\hat{p}_{i}, D_{S}\left[\hat{p}_{i+2}\right]\left(\hat{p}_{i+1}\right)\right)
$$

Similar to the proof of the Hamiltonian operator, to prove that $\hat{\mathfrak{A}}^{-1 *} \hat{\mathfrak{I}} \hat{\mathfrak{A}}^{-1}$ is symplectic, it is enough to show that the operators $\hat{\mathfrak{A}}^{-1 *} S_{i} \hat{\mathfrak{A}}^{-1}$ for $i=0,1,2$ are symplectic. These can be found in [3].

ACKNOwLEdgements. The authors thank Sasha Mikhailov and an unknown referee for pointing out a simplified form of the resulting evolution equation.

\section{REFERENCES}

1. S. C. Anco, Group-invariant soliton equations and bi-Hamiltonian geometric curve flows in Riemannian symmetric spaces, J. Geom. Phys. 58 (2008), 1-37.

2. A. Arvanitoyeorgos, An introduction to Lie groups and the geometry of homogeneous spaces, volume 22 of Student Mathematical Library. American Mathematical Society, Providence, RI, 2003. Translated from the 1999 Greek original and revised by the author.

3. E. Asadi, Integrable system in symplectic geometry, $\mathrm{PhD}$ thesis (vrije Universiteit Amsterdam, 2008).

4. A. Doliwa and P. M. Santini, An elementary geometric characterization of the integrable motions of a curve, Phys. Lett. A, 185 (4) (1994), 373-384.

5. I. Dorfman, Dirac structures and integrability of nonlinear evolution equations, in Nonlinear science: Theory and applications (John Wiley \& Sons Ltd., Chichester, 1993).

6. R. Hasimoto, A soliton on vortex filament, J. Fluid Mechanics, 51 (1972), 477-485. 
7. Sigurdur Helgason, Differential geometry, Lie groups, and symmetric spaces, in Pure and applied mathematics, vol 80 (Academic Press Inc., New York, 1978).

8. Y. Kosmann-Schwarzbach and F. Magri, Lax-Nijenhuis operators for integrable systems, J. Math. Phys., 37 (12) (1996), 6173-6197.

9. F. Magri, C. Morosi and O. Ragnisco, Reduction techniques for infinite-dimensional Hamiltonian systems: some ideas and applications, Comm. Math. Phys., 99 (1) (1985), 115-140.

10. F. Magri and C. Morosi, On the reduction theory of the Nijenhuis operators and its applications to Gel'fand-Dikil equations, in: IUTAM-ISIMM symposium on modern developments in analytical mechanics, Vol. II (Proc. Sympos. Torino, 1982), (1983), 599-626.

11. G. Marí Beffa, J. A. Sanders and J. P. Wang, Integrable systems in 3-dimensional Riemannian geometry, J. Nonlinear Sci., 12 (2) (2002), 143-167. 1986).

12. P. J. Olver, Applications of Lie groups to Differential Equations (Springer, New York,

13. Jan A. Sanders and Jing Ping Wang, Integrable systems in $n$-dimensional Riemannian geometry, Mosc. Math. J., 3 (4) (2003), 1369-1393.

14. Jan A. Sanders and Jing Ping Wang, Integrable systems in $n$-dimensional conformal geometry, J. Difference Equ. Appl., 12 (10) (2006), 983-995.

15. R. W. Sharpe, Differential geometry, in Graduate Texts in Mathematics, vol. 166 (Springer-Verlag, New York, 1997). Cartan's generalization of Klein's Erlangen program, With a foreword by S. S. Chern.

16. Chuu-Lian Terng, Soliton equations and differential geometry, J. Differential Geom., 45 (2) (1997), 407-445.

17. Chuu-Lian Terng and Gudlaugur Thorbergsson, Completely integrable curve flows on adjoint orbits. Results Math., 40(1-4) (2001) 286-309. (Dedicated to Shiing-Shen Chern on his 90th birthday.)

18. Chuu-Lian Terng and Karen Uhlenbeck, Schrödinger flows on Grassmannians, in Integrable systems, geometry, and topology, vol. 36 of AMS/IP Stud. Adv. Math., Amer. Math. Soc., Providence, RI, (2006), 235-253. 